\title{
Article
}

\section{Facilitating Versus Inhibiting the Transmission of Drug Abuse from High-Risk Parents to Their Children: A Swedish National Study}

\author{
Kenneth S. Kendler ${ }^{1,2}$, Henrik Ohlsson ${ }^{3}$, Jan Sundquist ${ }^{3,4,5}$ and Kristina Sundquist ${ }^{3,4,5}$ \\ ${ }^{1}$ Virginia Institute for Psychiatric and Behavioral Genetics, Virginia Commonwealth University, Richmond, VA, USA, ${ }^{2}$ Department of Psychiatry, Virginia \\ Commonwealth University, Richmond, VA, USA, ${ }^{3}$ Center for Primary Health Care Research, Lund University, Malmö, Sweden, ${ }^{4}$ Department of Family Medicine and \\ Community Health, Department of Population Health Science and Policy, Icahn School of Medicine at Mount Sinai, New York, NY, USA and ${ }^{5}$ Center for \\ Community-Based Healthcare Research and Education (CoHRE), Department of Functional Pathology, School of Medicine, Shimane University, Matsue, Japan
}

\begin{abstract}
We seek to identify factors that facilitate or inhibit transmission of drug abuse (DA) from high-risk parents to their children. In 44,250 offspring of these parents, ascertained from a Swedish national sample for having a mother and/or father with DA, we explored, using Cox models, how the prevalence of DA was predicted by potentially malleable risk factors in these high-risk parents, their spouses and the rearing environment they provided. Analyses of offspring of discordant high-risk siblings and offspring of discordant sibling-in-laws and step-parents aided causal inference. Risk for DA in the children was associated with high-risk and married-in parental externalizing psychopathology, a range of other features of these parents (e.g., low education and receipt of welfare), and aspects of the rearing environment (e.g., neighborhood deprivation and number of nearby drug dealers). Offspring of discordant high-risk siblings, siblings-in-laws and step-parents suggested that nearly all these associations were partly causal. A multivariate analysis utilizing offspring of discordant high-risk siblings identified the six most significant potentially malleable risk factors for offspring DA: (1) criminal behavior (CB) in married-in parent, (2) community peer deviance, (3) broken family, (4) DA in high-risk parent, (5) CB in high-risk parent and (6) number of family moves. Children in the lowest decile of risk had a 50\% reduction in their DA prevalence, similar to that seen in the general population. We conclude that transmission of DA from high-risk parents to children partly results from a range of potentially malleable risk factors that could serve as foci for intervention.
\end{abstract}

Keywords: Drug abuse; Sweden; parent-offspring transmission

(Received 22 October 2019; accepted 23 October 2019; First Published online 3 February 2020)

Drug abuse (DA) is strongly transmitted within families (Bierut et al., 1998; Marmorstein et al., 2009; Merikangas et al., 1998) as a result of both genetic- and familial-environmental effects (Kendler et al., 2003, 2016, 2017, 2019; Kendler, Maes et al., 2013; Kendler, Ohlsson et al., 2013; Tsuang et al., 1996). The familial transmission of substance use disorders is typically studied to clarify the etiology of DA. In this article, we examine it as a process upon which we seek to find ways to intervene. While theoretically possible, examining these questions by controlled experimentation is unlikely to be feasible. Instead, using the extensive data available to us in the Swedish registries, we have tried to address this question in an epidemiological framework at the descriptive and hopefully also at the causal level.

We begin by defining a high-risk parent (individual II-3 in Figure 1) whose high-risk status is operationalized by having a father or mother with DA (individual I-2). We examine what features of this high-risk parent and his/her spouse (II-4)

Author for correspondence: Kenneth S. Kendler, Email: Kenneth.Kendler@vcuhealth.org Cite this article: Kendler KS, Ohlsson H, Sundquist J, and Sundquist K. (2020) Facilitating Versus Inhibiting the Transmission of Drug Abuse from High-Risk Parents to Their Children: A Swedish National Study. Twin Research and Human Genetics 23: 1-7, https://doi.org/10.1017/thg.2020.1 facilitate or inhibit the transmission of DA to their child (III-2). Put another way, for parents with a family history of DA, what distinguishes those who transmit the familial vulnerability to their children so that they likely go on to develop DA versus those who break the transmission pattern and raise unaffected children?

Because interventions are far better based on causally informative rather than solely descriptive findings, we implement three additional analyses. First, we utilize an offspring of discordant high-risk sibling design that compares risk factors for transmission of DA in cousins from high-risk full siblings discordant for that risk factor. For example, in clarifying whether criminal behavior $(\mathrm{CB})$ in parents truly increases risk for DA in their children, we compare the rates of DA in first cousins (individual III-1 vs. III-2) who are offspring of two high-risk siblings discordant for a criminal history (i.e., individuals II-2 and II-3). Second, we implement an offspring of discordant siblings-in-law design where the risk is compared in cousins who are the offspring of married-in spouses discordant for key risk factors (i.e., comparing risk in III-1 vs. III-2, given that II- 1 and II-4 are discordant). Third, to help us control for transmitted genetic effects from the second to third generation, we compare the impact of certain risk factors in biological versus step-parents in the second generation of our high-risk pedigrees. 


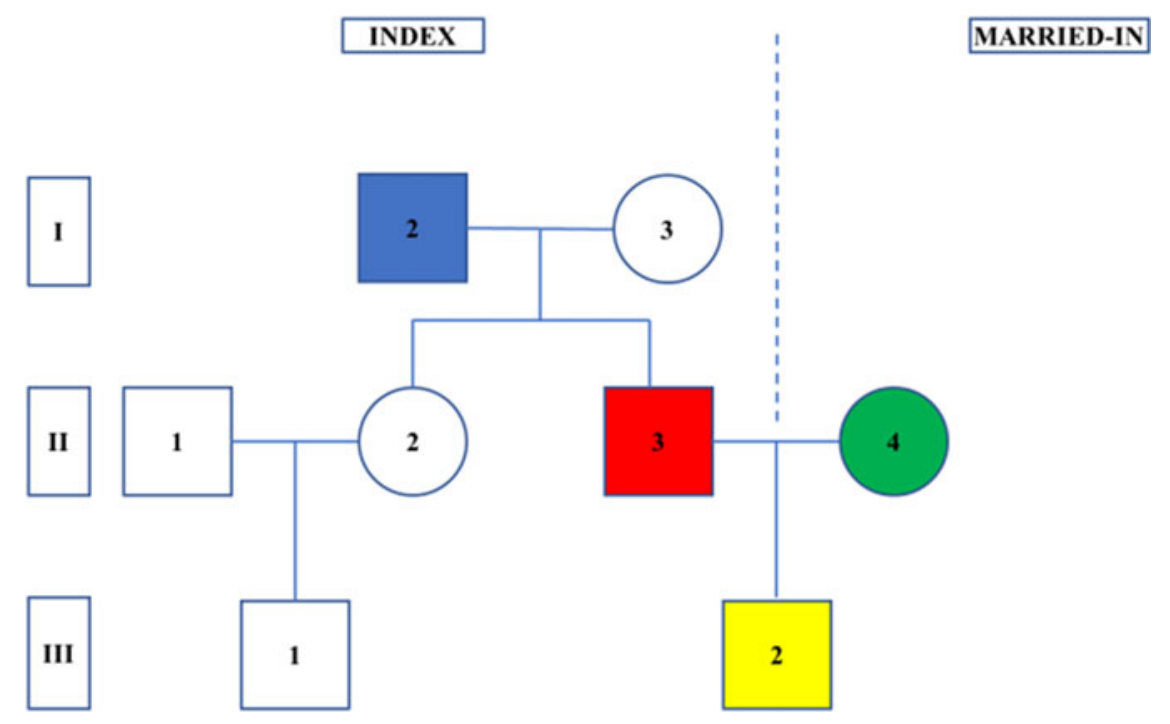

Fig. 1. A depiction of the pedigrees examined in this report. The Roman numerals on the left represent the generation: (1) grandparental, (2) parental and (3) grandchildren. The Arabic numerals within the circles (females) and squares (males) represent the individuals. Pedigrees were formed by selecting the children (in yellow, individual III-2) who had $\geq 1$ grandparent affected with drug abuse (in blue, individual I-2). Key individuals examined in our analyses are the high-risk parent (in red, individual I-3), his/her spouse (in green, individual I-4), high-risk aunts/uncles (individual II-1) and first cousins (individuals III-1). In this example, the sexes of the key individuals (I-2, II-3 and III-2) are set to male, but this choice is arbitrary and, in the analyses, can be either sex.

\section{Methods}

We used Swedish population-based registers with national coverage, linking them using each person's unique identification number. To preserve confidentiality, this ID number was replaced by a serial number. We secured ethical approval for this study from the Regional Ethical Review Board of Lund University (No. 2008/409).

From the registers, we selected all individuals, the children in our design, born from January 1, 1970 to December 31, 1990, who had at least one grandparent registered for DA $(n=45,837)$. In situations where both maternal and paternal grandparents were registered for DA, we randomly selected one of them as the index family $(n=942)$. In total, 1116 individuals either died or emigrated prior to age 15. Figure 1 illustrates the structure of the pedigrees included in the sample. For each child (III-2), we created their pedigree that included grandparents (I-2, -3) and parent (II-3) from the high-risk family as well as the parent (II-4) from the married-in family. For both parents of the child (II-3, -4), we included information on registrations of DA, alcohol use disorder (AUD) and $\mathrm{CB}$, psychiatric registrations, hospitalizations, educational status, resilience score, unemployment status, early retirement, social welfare and age at first child. We also included several variables that describe the home and community environment in which the proband was raised: broken family, peer deviance, number of drug dealers in the area, number of times the proband moved prior to age 15 and neighborhood deprivation score. In families where a step-parent was a part of the family $(n=4274)$, we also included information on DA, AUD, CB, psychiatric registrations, hospitalization, educational status, unemployment status and early retirement for the step-parent. For a definition of step-parent and the predictor variables, see Supplementary Table 1.

We first developed univariate Cox regression models for all potentially malleable risk factors for DA in the child. These are features of the high-risk parent, their spouse and the home situation in which the child was reared. Follow-up time, in number of months, was measured from age 15 until year of first registration for DA, death or emigration, whichever came first. Observations were right-censored at the end of follow-up (December 31, 2015). In all models, we also controlled for year of birth and sex of the child. Individuals were clustered within the same grandparent and parent, and we used a robust sandwich estimator to take this into account. We also checked the proportionality assumption using both the Schoenfeld residuals and an interaction term between time and the predictor of interest. No severe violations were found. For these analyses, we also employed an offspring of discordant sibling design; 5677 grandparents had two or more children who in turn had at least one child each and were eligible for these analyses which included 25,670 children. For features of the high-risk parent, this means that we are contrasting rates of DA in cousins with the same grandparent but parents with different levels of the predictor variables. The hazard ratio (HR) is then adjusted for the grandparental cluster and therefore accounts for an array of unmeasured genetic and environmental factors shared among parents of the cousins. For features of the home environment (i.e., peer deviance, number of drug dealers, number of moves and broken family), this means that we are contrasting rates of DA in cousins with the same grandparent but with different levels of the predictor variables (i.e., the probands are either cousins or full siblings). For features of the spouses, these analyses mean that we are contrasting rates of DA in cousins with the same grandparent but married-in parents with different levels of the predictor variables. We call these offspring of discordant siblings-in-law. Using univariate Cox regression models, we also investigated risk factors for DA in the proband in the step-parent sample.

We then fitted a forward stepwise Cox regression ( $p$ value for entry: .2 and $p$ value for stay: .1) using all the potentially malleable risk factors. To assess the relative importance of individual predictors in the models, we used the $t$-statistic for each model parameter using the Caret Package in R (Kuhn, 2008). In order to verify the selection process from the stepwise regression, we also used lasso regression. The results were similar: the four most influential 
Table 1. Risk factors for drug abuse in the child from potentially malleable risk factors in the high-risk and married-in parent, their rearing environment and from step-parents

\begin{tabular}{|c|c|c|c|c|c|c|c|}
\hline \multirow[b]{2}{*}{$\begin{array}{l}\text { Characteristics of high-risk and } \\
\text { married-in parent }\end{array}$} & \multicolumn{3}{|c|}{ High-risk parent } & \multicolumn{3}{|c|}{ Married-in parent } & \multirow[b]{2}{*}{ Step-parent } \\
\hline & Prevalence & $\begin{array}{l}\text { Raw HR } \\
95 \% \mathrm{Cls}\end{array}$ & $\begin{array}{l}\text { Co-sibling HR } \\
95 \% \mathrm{Cls}^{\mathrm{a}}\end{array}$ & Prevalence & $\begin{array}{l}\text { Raw HR } \\
95 \% \text { Cls }\end{array}$ & $\begin{array}{l}\text { Co-sibling-in-law } \\
\text { HR } 95 \% \mathrm{Cls}^{\text {b }}\end{array}$ & \\
\hline Drug abuse & $8.1 \%$ & $3.44[3.15,3.74]$ & $1.82[1.55,2.15]$ & $5.2 \%$ & $3.44[3.12,3.79]$ & $1.73[1.48,2.03]$ & $2.40[1.78,3.24]$ \\
\hline Alcohol use disorder & $14.2 \%$ & $2.57[2.38,2.77]$ & $1.45[1.26,1.67]$ & $11.7 \%$ & $2.65[2.45,2.87]$ & $1.70[1.50,1.93]$ & $1.67[1.36,2.06]$ \\
\hline Criminal behavior & $20.8 \%$ & $2.94[2.74,3.16]$ & $1.54[1.34,1.76]$ & $20.3 \%$ & $2.87[2.68,3.08]$ & $1.78[1.58,2.00]$ & $1.29[1.08,1.54]$ \\
\hline $\begin{array}{l}\text { Psychiatric registration } \\
\text { (all registers) } \geq 1\end{array}$ & $41.6 \%$ & $1.97[1.84,2.11]$ & $1.58[1.40,1.79]$ & $33.5 \%$ & $1.78[1.66,1.90]$ & $1.45[1.31,1.61]$ & $1.59[1.35,1.89]$ \\
\hline Hospitalization (any) $\geq 1$ & $20.9 \%$ & $1.43[1.32,1.55]$ & $1.23[1.07,1.41]$ & $20.4 \%$ & $1.26[1.16,1.37]$ & $1.23[1.09,1.38]$ & $1.24[1.01,1.51]$ \\
\hline Low education $-z$ score & $0.17(0.93)$ & $1.41[1.35,1.48]$ & $1.17[1.08,1.27]$ & $0.1(0.9) \%$ & $1.44[1.38,1.51]$ & $1.22[1.13,1.31]$ & $1.04[0.93,1.15]$ \\
\hline $\begin{array}{l}\text { Unemployment at age } 45 \text { or } 55 \\
\text { (yes/no) }\end{array}$ & $21.0 \%$ & $1.63[1.52,1.76]$ & $1.10[0.97,1.26]$ & $19.5 \%$ & $1.68[1.56,1.81]$ & $1.25[1.12,1.40]$ & $1.05[0.85,1.30]$ \\
\hline $\begin{array}{l}\text { Early retirement at age } 45 \text { or } 55 \\
\text { (yes/no) }\end{array}$ & $16.0 \%$ & $1.89[1.75,2.05]$ & $1.33[1.16,1.52]$ & $12.0 \%$ & $1.73[1.59,1.89]$ & $1.24[1.09,1.42]$ & $1.47[1.14,1.89]$ \\
\hline $\begin{array}{l}\text { Social welfare at age } 45 \text { or } 55^{c} \\
\text { (yes/no) }\end{array}$ & $13.0 \%$ & $2.97[2.76,3.21]$ & $1.83[1.59,2.11]$ & - & & & - \\
\hline $\begin{array}{l}\text { Young age at first child } \\
\text { (prior to age 20) }\end{array}$ & $12.7 \%$ & $2.10[1.93,2.30]$ & $1.14[0.98,1.33]$ & $8.7 \%$ & $1.91[1.72,2.11]$ & $1.10[0.95,1.28]$ & - \\
\hline \multicolumn{8}{|l|}{$\begin{array}{l}\text { Home situation in which child is } \\
\text { raised }\end{array}$} \\
\hline $\begin{array}{l}\text { Neighborhood deprivation } z \text { score } \\
\text { (age } 45 \text { or } 55 \text { ) }\end{array}$ & $0.4(1.5)$ & $1.20[1.18,1.22]$ & $1.08[1.04,1.11]$ & & & & \\
\hline $\begin{array}{l}\text { Peer deviance at age } 15 \text { of child } \\
\text { (\% units) }\end{array}$ & $3.9(2.8)$ & $1.06[1.05,1.08]$ & $1.05[1.03,1.06]$ & & & & \\
\hline Drug dealers at age 15 ( $z$ score) & $0.5(1.6)$ & $1.13[1.12,1.15]$ & $1.06[1.04,1.09]$ & & & & \\
\hline $\begin{array}{l}\text { Number of moves } \\
\text { (between ages } 0 \text { and } 15 \text { of child) }\end{array}$ & $1.8(1.8)$ & $1.25[1.23,1.27]$ & $1.12[1.09,1.14]$ & & & & \\
\hline Broken family & $52.1 \%$ & $3.30[3.06,3.56]$ & $1.78[1.59,1.99]$ & & & & \\
\hline \multicolumn{8}{|l|}{ Control variables } \\
\hline Year of birth grandchild & $1983(5.9)$ & $1.10[1.09,1.11]$ & $1.08[1.07,1.09]$ & & & & \\
\hline Sex grandchild (males) & $51.7 \%$ & $2.22[2.08,2.38]$ & $2.23[2.06,2.42]$ & & & & \\
\hline
\end{tabular}

Note: Controlled for year of birth and sex in the third generation (grandchildren).

${ }^{a}$ Offspring of discordant high-risk siblings.

boffspring of discordant high-risk siblings-in-law.

'Social welfare is assigned to a household not to individual parents.

variables were identical, and five out of six variables dropped were identical. We thereafter fitted a multivariate model with the top six most important variables. For these analyses, we also employed an offspring of discordant sibling analysis. As this analysis compares probands with the same grandparent, the number of possible predictor variables included in the analysis is limited. We chose six as we were still able to get stable estimates for the offspring of discordant sibling analysis. We chose this cutoff because, as outlined in Supplementary Table 2, the seventh most important variable had a much lower $t$-statistic. Finally, we fitted a multivariate logistic regression model (using the variables from the stepwise Cox regression model) to a random half as a training sample. Results from that model were then applied to the second random half and were evaluated using a receiver operating characteristic (ROC) curve. We also created a risk score based on the nine groups of the predicted probabilities and used it as a predictor variable in a Cox regression model. We then sought to assess the degree to which the association between the risk score and DA in proband reflects confounding by familial risk factors using the offspring of discordant sibling design. Using a stratified model with a separate stratum for each index-grandparent, we refitted the model. Within each stratum, the HR for the risk score was adjusted for unmeasured shared genetic and environmental factors among probands to the same grandparent. The regression models were performed using SAS 9.4 (SAS Institute, 2012).

\section{Results}

\section{Descriptive Statistics}

We studied 44,250 children of high-risk parents of whom $51.8 \%$ were male. We followed them from age 15 to a mean age (SD) at the end of follow-up of 32.4 (5.9). DA was uncommon in the grandparental generation with a prevalence $( \pm 95 \% \mathrm{CIs})$ of $0.8 \%$ $[0.8,0.8]$. The prevalence of DA in our high-risk parents, $8.1 \%$ $[7.9,8.3]$, was increased approximately 3.5 -fold, over the general population: $2.2 \%[2.2,2.2]$. Parallel results for our children were $8.9 \%[8.7,9.1]$ versus $4.7 \%[4.7,4.7]$. 
Table 2. Results of multivariate prediction of risk for drug abuse in the child from potentially malleable risk factors in the high-risk and married-in parent and their rearing environment calculated at a population and within-family (i.e., high-risk co-sibling control) level

\begin{tabular}{|c|c|c|c|}
\hline & Population multivariate & $\begin{array}{l}\text { Offspring of discordant } \\
\text { siblings multivariate }\end{array}$ & $\begin{array}{l}t \text {-Statistics from offspring } \\
\text { of discordant siblings model }\end{array}$ \\
\hline Broken family & $3.30[3.06,3.56]$ & $1.78[1.59,1.99]$ & 8.5 \\
\hline Broken family & $2.80[2.60,3.02]$ & $1.60[1.43,1.79]$ & 7.7 \\
\hline Criminal behavior - Parent & $2.36[2.21,2.52]$ & $1.48[1.30,1.69]$ & 5.6 \\
\hline Broken family & $2.34[2.17,2.53]$ & $1.50[1.34,1.68]$ & 6.5 \\
\hline Criminal behavior - High-risk parent & $2.18[2.04,2.33]$ & $1.50[1.32,1.71]$ & 5.8 \\
\hline Criminal behavior - Married-in parent & $2.09[1.96,2.24]$ & $1.60[1.43,1.79]$ & 8.1 \\
\hline Broken family & $2.26[2.09,2.44]$ & $1.46[1.30,1.63]$ & 6.2 \\
\hline Criminal behavior - High-risk parent & $2.12[1.98,2.26]$ & $1.46[1.28,1.67]$ & 5.5 \\
\hline Criminal behavior - Married-in parent & $2.03[1.90,2.18]$ & $1.58[1.41,1.76]$ & 8.0 \\
\hline Peer deviance at age 15 of child (\% units) & $1.05[1.04,1.06]$ & $1.05[1.04,1.07]$ & 5.2 \\
\hline Broken family & $2.16[2.00,2.34]$ & $1.43[1.27,1.60]$ & 5.8 \\
\hline Criminal behavior - High-risk parent & $1.85[1.72,1.99]$ & $1.34[1.17,1.54]$ & 4.2 \\
\hline Criminal behavior - Married-in parent & $1.96[1.83,2.10]$ & $1.55[1.39,1.74]$ & 7.8 \\
\hline Peer deviance at age 15 of child (\% units) & $1.05[1.04,1.06]$ & $1.05[1.04,1.07]$ & 5.2 \\
\hline Drug abuse — High-risk parent & $1.70[1.56,1.85]$ & $1.49[1.26,1.76]$ & 4.7 \\
\hline Broken family & $1.97[1.81,2.14]$ & $1.39[1.23,1.56]$ & 4.9 \\
\hline Criminal behavior - High-risk parent & $1.79[1.66,1.92]$ & $1.34[1.17,1.53]$ & 4.0 \\
\hline Criminal behavior - Married-in parent & $1.88[1.76,2.02]$ & $1.54[1.38,1.72]$ & 7.5 \\
\hline Peer deviance at age 15 of child (\% units) & $1.05[1.04,1.06]$ & $1.05[1.04,1.07]$ & 5.2 \\
\hline Drug abuse - High-risk parent & $1.66[1.53,1.81]$ & $1.48[1.25,1.75]$ & 4.6 \\
\hline Number of moves (between ages 0 and 15 of child) & $1.07[1.05,1.09]$ & $1.02[1.00,1.05]$ & 3.1 \\
\hline
\end{tabular}

\section{Potentially Malleable Risk Factors — Univariate Analyses}

Table 1 contains results for three sets of potentially malleable risk factors for DA in the children. These reflect features of (1) the high-risk parent, (2) their spouse and (3) the home situation in which their child was reared. For these analyses, we also present results of analyses of offspring of discordant high-risk siblings and of siblings-in-law.

DA in either the high-risk parent or spouse has a strong and similar impact on risk for DA in their child $(\mathrm{HR}=3.44)$. The risk is moderately lower with $\mathrm{CB}$ and AUD in the parents (HRs $\sim 2.9$ and 2.7, respectively). The HRs are appreciably smaller in the offspring of discordant high-risk sibling analyses (e.g., 1.82 for DA and 1.54 for $\mathrm{CB}$ ) but much larger than unity, suggesting a causal effect of these disorders on risk for DA in their child. A similar pattern is seen in the offspring of discordant siblingsin-law.

We then examine a range of other characteristics of the high-risk and married-in parents that might impact on risk for DA in the child. Focusing first on the high-risk parent, all the putative risk factors significantly predict child risk with HRs, ranging from 1.41 per standard deviation of lower education to 2.97 for the family being on social welfare. Results are similar for the married-in spouse. For the offspring of discordant high-risk sibling analyses, five of the seven associations are statistically significant (all but unemployment and young age at first child), although the associations are consistently weaker than the population-based analyses. A similar pattern is seen with the offspring of discordant siblings-in-law analyses.

We next explore the impact on child risk for DA risk on five features of the rearing environment. All were statistically significant, although the effect sizes were modest, except for a broken home which had the largest observed HR in our analyses: 3.30. In the offspring of discordant sibling analyses, all the HRs were lower but remained significant with the largest $(H R=1.78)$ seen for a broken home.

Finally, 9.5\% of the parent-offspring families contained a step-parent $(n=4274)$. Having step-parents with DA, AUD or CB significantly increased the risk for DA in their step-child. Of the five other parental features available in these step-parents, three were significantly associated with risk of DA in the child: psychiatric registration, hospitalization and early retirement.

\section{Multivariate Analysis of Malleable Factors}

Focusing on those features of the parental generation that were potentially malleable, we then combined them in a multiple regression to examine their aggregate predictive effect on DA in the proband - their offspring. The results are seen in Supplementary Table 2 with the effect size of each variable that remained in the predictive model indexed by its associated $t$-statistic. The six strongest predictors, in order of their effect size, were nonintact family, $\mathrm{CB}$ in the index parent, $\mathrm{CB}$ in the marriedin-parent, peer deviance in the community of residence, DA in the 


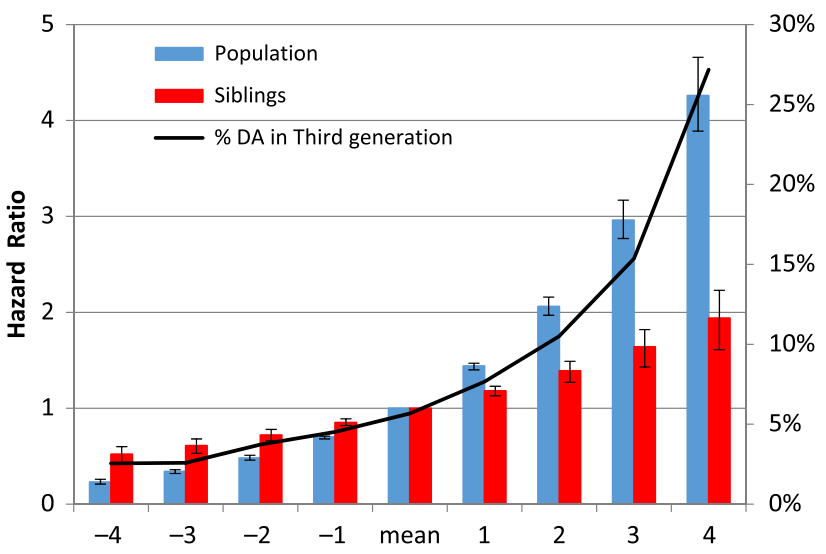

Fig. 2. Results of multivariate prediction of risk for drug abuse in the child from potentially malleable risk factors in the high-risk and married-in parent and their rearing environment calculated at the population and within-sibling levels and divided into nine risk categories.

index parent and number of moves of the nuclear family. ROC analyses of these measures in predicting offspring DA had an area under the curve of 0.74 (0.73-0.75; Supplementary Figure 1). We then divided this risk score into nine approximately equal groups (Figure 2). Looking within the entire population, compared to the median group, the HRs for DA in the offspring of the lowest and highest risk families were $0.23(0.21 ; 0.26)$ and $4.26(3.89 ; 4.66)$, respectively.

We then applied a co-sibling design to this same risk score with the results also depicted in Figure 2. The effects were more modest but likely to reflect largely causal effects with the lowest and highest groups relative to the median group now having HRs for DA of $0.52(0.44 ; 0.61)$ and $1.94(1.65 ; 2.27)$, respectively.

Finally, we examined, in Table 2, the predictive power in an offspring of discordant sibling design, for the six most potent risk factors in our multivariate model. We focus on the last model in the table, which shows that, taken together, each remained a significant predictor of risk for DA in our pedigree proband (\# III-2) compared to his/her cousin (\# III-1) when the two siblings (II-2 and II-3) are discordant for the given trait. In order of effect size (from largest to smallest), as indexed by their $t$ value, the predictive power of the six variables was (1) CB in married-in spouse, (2) community peer deviance, (3) broken family, (4) DA in the index parent, (5) $\mathrm{CB}$ in the index parent and (6) number of moves in the family.

\section{Discussion}

We defined high-risk parents as having a father or mother affected with DA - a rare diagnosis in that generation. We demonstrated that a wide range of specific features of both the high-risk parent and their marital partner predicted risk for DA in their children. Risk factors included their own history of DA, CB, AUD and other psychiatric illness as well as their educational level, employment history and receipt of social welfare. Critically, when examined using discordant high-risk sibling and sibling-in-law models, most predictors remained significant although diminished in effect size.

A similar pattern was observed for the five measures of rearing environment. All significantly predicted risk for DA in the offspring, and all remained significant in the discordant sibling and sibling-in-law models. These results suggest that a meaningful proportion of the association of all these risk factors to DA risk in the children is likely causal.

We also examined risk in the offspring of step-parents in these families. Having a step-parent with DA, CB and AUD, psychiatric illness, hospitalization or on social welfare significantly increased risk for DA in the children. These results are important in providing additional evidence that the impact of the putative risk factors assessed in parents cannot be entirely explained by genetic effects. Rather, the risk was, at least in part, transmitted environmentally to their child.

In considering interventions to reduce risk for DA in the offspring of a high-risk parent, we have identified six risk factors which at least theoretically could be modified by that parent: (1) avoid marrying a spouse who develops $\mathrm{CB},(2)$ raise a child in a community where few if any of his/her peers go on to develop DA, (3) maintain an intact family while raising the child, (4) and (5) avoid yourself developing DA or CB and (6) do not move frequently while raising your child.

Noteworthy is the diversity of these potentially malleable factors. Of the top six, two reflect the high-risk parent avoiding high-risk outcomes themselves and one each reflects choice of spouse, community of residence when child-rearing, intactness of the family unit and the history of disruptions due to family moves. Our results mirror the diverse nature of the risk factors for the complex phenotype of DA. Of particular interest, our results suggest that, even if only theoretical, if high-risk parents were able to achieve most of these goals, the chances that their child would develop DA would be reduced by approximately $50 \%$, which would be close to baseline rate expectations in the population. That is, by taking these steps, the parent could effectively wipe out the impact of their familial risk for DA on their children's probability to develop DA.

Our findings are consistent with prior evidence that changes in parental behavior can impact causally on child psychopathology. Using a natural experiment, Costello et al. (2003) showed that receipt of additional family income permitted parents to supervise better their children's behavior, resulting in a significant reduction of externalizing symptoms. Weissman et al. (2006) showed that effective treatment of depressive symptoms in mothers in a controlled trial resulted in improvement in their children's psychopathology. We have shown that a diagnosis of DA in adoptive and step-parents significantly increases risk for DA in their offspring (Kendler et al., 2017) and that, in a matched control design, onset of DA in a parent is, over the next 3 years, associated with a significantly increased risk of DA onset in his offspring (Kendler et al., 2019). Consistent with our findings here from our co-sibling analyses, these results demonstrate that DA can be psychologically transmitted across generations. Furthermore, adoptees exposed to the high-quality rearing environment provided by screened adoptive parents had substantially lower rates of DA compared to their full or half-siblings raised by their biological parents (Kendler et al., 2016).

Multiple family-based programs seeking to prevent DA have been proposed and widely evaluated (Kumpfer, 2002; Kumpfer \& Alvarado, 2003; Ladis et al., 2019; Lochman \& van den Steenhoven, 2002) with reviews supporting the efficacy of several approaches including parent skills training, parent engagement, family therapy and in-home family support. Our results are complementary to this literature and arise from a different empirical framework. We would hope for points of contact between this large intervention literature and the naturalistic approach we have taken to the study of familial transmission of DA risk. 
Unlike the family-based interventions, which typically employ only short follow-up periods and examine substance use as their main outcome, our study follows our sample of probands over a mean of 17 years and examines the more clinical relevant outcome of DA.

\section{Limitations}

These results should be interpreted in the context of five potentially significant limitations. First, our results are limited to Sweden and may or may not extrapolate to different types of populations. Second, our ascertainment of DA, AUD and $\mathrm{CB}$ was based on registries. Registration for DA and AUD is likely to assess the more severe end of the clinical spectrum but is validated by a strong level of agreement between different sources of registration - medical, criminal and pharmacy based (Kendler et al., 2012, 2015).

Third, our analyses did not control for differences in the age of our high-risk parents and siblings discordant for the risk factor of interest. Large age differences might reduce the quality of the environmental matching undermining our attempts at causal inference. We repeated these analyses restricting these sibling pairs to those born within 5 years of each other. As seen in Supplementary Table 3, the results did not change appreciably.

Fourth, causal inference from nonexperimental data is always challenging (Glass et al., 2013). We have used three methods to provide insight into causal effects, but each has limitations. Our high-risk co-sibling analyses control for overall familial background, but siblings share only $50 \%$ of their genes identical by descent, and siblings-in-law are likely well matched for social background but are typically not genetically related. Thus, while our estimates of causal effects using our co-sibling designs are only approximate and are surely more accurate than the raw associations, they are more likely to over- than underestimate causal effects.

Finally, directly intervening on a number of the risk factors that we identified in our epidemiological analyses to reduce the probability of high-risk individuals transmitting DA to their children would be very challenging. We do not intend to claim that there is a direct or easy link between active prevention methods and any of our findings. However, our results serve a useful didactic purpose in showing that such interventions are theoretically possible and that the familial transmission of DA is not an inevitable process immune to prevention efforts.

\section{Conclusions}

Given the relatively high heritability of DA (Kendler et al., 2003; Kendler, Maes et al., 2013; Tsuang et al., 1996), some might conclude that interventions aimed at high-risk parents seeking to reduce rates of DA in their children would likely be unsuccessful. However, given substantial prior evidence for environmental transmission of DA within families (Kendler et al., 2016, 2017, 2019; Kendler, Ohlsson et al., 2013), for which interventions could plausibly be effective, we sought to rigorously address this question. Our results are encouraging. Using descriptive and causally informative analytic approaches, we showed that risk factors for children in high-risk parents are diverse and all are in part the result of causal effects. That is, for a high-risk parent, developing DA, living in a rough neighborhood, marrying an at-risk individual and doing poorly in their employment transmit risk to the child above and beyond the genes transmitted to them. Our multivariate model, the validity of which is supported by our results with step-parents, suggests that intervening on the most important modifiable risk factors could reduce the rate of DA in children by about $50 \%$. Given the rapid advances in our understanding of the molecular genetic basis of DA, we should not lose sight of the contributions of environmental factors to its familial transmission and the potential to use our increasing knowledge of these effects to improve our targeted interventions.

Supplementary material. To view supplementary material for this article, please visit https://doi.org/10.1017/thg.2020.1.

Financial support. This project was supported by grant R01DA030005 from the National Institutes of Health, NIAAA, grants from the Swedish Research Council (2016-01176), as well as Avtal om Läkarutbildning och Forskning funding from Region Skåne.

\section{Conflicts of interest. None.}

Ethical standards. The authors assert that all procedures contributing to this work comply with the ethical standards of the relevant national and institutional committees on human experimentation and with the Helsinki Declaration of 1975 , as revised in 2008 . We secured ethical approval for this study from the Regional Ethical Review Board of Lund University (No. 2008/409).

Informed consent. As approved by Swedish ethical authorities, informed consent was not obtained from individual participants included in this study.

\section{References}

Bierut, L. J., Dinwiddie, S. H., Begleiter, H., Crowe, R. R., Hesselbrock, V., Nurnberger, J. I., Jr. ... Reich, T. (1998). Familial transmission of substance dependence: Alcohol, marijuana, cocaine, and habitual smoking: A report from the Collaborative Study on the Genetics of Alcoholism. Archives of General Psychiatry, 55, 982-988.

Costello, E. J., Compton, S. N., Keeler, G., \& Angold, A. (2003). Relationships between poverty and psychopathology - A natural experiment. Journal of the American Medical Association, 290, 2023-2029.

Glass, T. A., Goodman, S. N., Hernan, M. A., \& Samet, J. M. (2013). Causal inference in public health. Annual Review of Public Health, 34, 61-75.

Kendler, K. S., Jacobson, K. C., Prescott, C. A., \& Neale, M. C. (2003). Specificity of genetic and environmental risk factors for use and abuse/ dependence of cannabis, cocaine, hallucinogens, sedatives, stimulants, and opiates in male twins. American Journal of Psychiatry, 160, 687-695.

Kendler, K. S., Ji, J., Edwards, A. C., Ohlsson, H., Sundquist, J., \& Sundquist, K. (2015). An extended Swedish national adoption study of alcohol use disorder. JAMA Psychiatry, 72, 211-218.

Kendler, K. S., Maes, H. H., Sundquist, K., Ohlsson, H., \& Sundquist, J. (2013). Genetic and family and community environmental effects on drug abuse in adolescence: A Swedish national twin and sibling study. American Journal of Psychiatry, 171, 209-217.

Kendler, K. S., Ohlsson, H., Sundquist, J., \& Sundquist, K. (2019). A contagion model for within-family transmission of drug abuse. American Journal of Psychiatry, 176, 239-248.

Kendler, K. S., Ohlsson, H., Sundquist, K., \& Sundquist, J. (2013). Withinfamily environmental transmission of drug abuse: A Swedish national study. JAMA Psychiatry, 70, 235-242.

Kendler, K. S., Ohlsson, H., Sundquist, K., \& Sundquist, J. (2016). The rearing environment and risk for drug abuse: a Swedish national high-risk adopted and not adopted co-sibling control study. Psychological Medicine, 46, 1359-1366.

Kendler, K. S., Ohlsson, H., Sundquist, K., \& Sundquist, J. (2017). Sources of parent-child transmission of drug abuse: Path analyses of not-lived-with parental, stepparental, triparental, and adoptive families. Journal of Nervous and Mental Disorders, 206, 239-244. 
Kendler, K. S., Sundquist, K., Ohlsson, H., Palmer, K., Maes, H., Winkleby, M. A., \& Sundquist, J. (2012). Genetic and familial environmental influences on the risk for drug abuse: A national Swedish adoption study. Archives of General Psychiatry, 69, 690-697.

Kuhn, M. (2008). Building predictive models in R using the Caret package. Journal of Statistical Software, 28, 1-26.

Kumpfer, K. L. (2002). Prevention of alcohol and drug abuse: What works? Substance Abuse, 23, 23-45.

Kumpfer, K. L., \& Alvarado, R. (2003). Family-strengthening approaches for the prevention of youth problem behaviors. American Psychology, 58, 457-465.

Ladis, B. A., Macgowan, M., Thomlison, B., Fava, N. M., Huang, H., Trucco, E. M., \& Martinez, M. J. (2019). Parent-focused preventive interventions for youth substance use and problem behaviors: A systematic review. Research on Social Work Practice, 29, 420-442.

Lochman, J. E., \& van den Steenhoven, A. (2002). Family-based approaches to substance abuse prevention. Journal of Primary Prevention, 23, 49-114.
Marmorstein, N. R., Iacono, W. G., \& McGue, M. (2009). Alcohol and illicit drug dependence among parents: Associations with offspring externalizing disorders. Psychological Medicine, 39, 149-155.

Merikangas, K. R., Stolar, M., Stevens, D. E., Goulet, J., Preisig, M. A., Fenton, B., \& Rounsaville, B. J. (1998). Familial transmission of substance use disorders. Archives of General Psychiatry, 55, 973-979.

SAS Institute Inc. (2012). SAS/STAT ${ }^{\circledast}$ online documentation, version 9.4. Cary, NC: SAS Institute.

Tsuang, M. T., Lyons, M. J., Eisen, S. A., Goldberg, J., True, W., Meyer, J. M., \& Eaves, L. J. (1996). Genetic influences on abuse of illicit drugs: A study of 3,297 twin pairs. American Journal of Medical Genetics, 67, 473-477.

Weissman, M. M., Pilowsky, D. J., Wickramaratne, P. J., Talati, A., Wisniewski, S. R., Fava, M. \& STAR *D-Child Team (2006). Remissions in maternal depression and child psychopathology: A STAR*D-child report. Journal of the American Medical Association, 295, 1389-1398. 